\title{
Neutrinos and ultra-high-energy nuclei from blazars: from a single-source model to a population study
}

\section{Xavier Rodrigues*}

Deutsches Elektronen-Synchrotron (DESY), Platanenallee 6, D-15738 Zeuthen, Germany

E-mail: xavier.rodrigues@desy.de

\section{Anatoli Fedynitch}

Deutsches Elektronen-Synchrotron (DESY), Platanenallee 6, D-15738 Zeuthen, Germany

E-mail: anatoli.fedynitchedesy.de

\section{Shan Gao}

Deutsches Elektronen-Synchrotron (DESY), Platanenallee 6, D-15738 Zeuthen, Germany E-mail: shan.gaoddesy.de

\section{Andrea Palladino}

Deutsches Elektronen-Synchrotron (DESY), Platanenallee 6, D-15738 Zeuthen, Germany E-mail: andrea.palladino@desy.de

\section{Denise Boncioli}

Deutsches Elektronen-Synchrotron (DESY), Platanenallee 6, D-15738 Zeuthen, Germany Gran Sasso Science Institute (GSSI), Viale Francesco Crispi 7, 67100 L'Aquila, Italy INFN, Laboratori Nazionali del Gran Sasso (LNGS), 67100 Assergi, L'Aquila, Italy E-mail: denise.boncioli@desy.de

\section{Walter Winter}

Deutsches Elektronen-Synchrotron (DESY), Platanenallee 6, D-15738 Zeuthen, Germany E-mail: walter.winter@desy.de

Five years after the discovery of the diffuse astrophysical neutrino flux, its origin is still unclear. At the same time, composition measurements of ultra-high-energy cosmic rays (UHECRs) seem to indicate a component heavier than protons. In this talk we present an interaction model of protons and heavier nuclei in blazar jets. In bright blazars, photo-nuclear interactions lead to extensive nuclear cascades of lighter elements, and we calculate numerically for the first time their effect on blazar neutrino emission. In the case of flat-spectrum radio quasars (FSRQs), our model considers the effect of CR propagation in the broadline region, which boosts neutrino production in these sources. We then apply this model to the entire cosmological distribution of blazars using the updated blazar sequence, based on a large sample from the Fermi 3LAC catalog. We conclude that the IceCube flux of sub-PeV and $\mathrm{PeV}$ neutrinos can be explained by a population of low-luminosity blazars, not yet observed in gamma rays but predicted by cosmological evolution models. On the other hand, the IceCube stacking analysis limits the contribution from bright FSRQs, which places constraints on their hadronic content. Based on this model, we can estimate the number of future coincident neutrino detections like that of TXS 0506+056.

36th International Cosmic Ray Conference -ICRC2019-

July 24th - August 1st, 2019

Madison, WI, U.S.A. 


\section{Introduction}

High-energy astrophysical neutrinos, recently detected by IceCube [1,2], are the smokinggun signature of hadronic interactions by ultra-high-energy cosmic rays (UHECRs). However, the astrophysical objects where these processes take place remain unknown. Blazars, the sub-class of active galactic nuclei (AGN) whose relativistic jet points towards Earth, have long been proposed as promising neutrino emitters given their bright emission across the electromagnetic spectrum and strong temporal variability.

Current lepto-hadronic blazar models explain neutrino emission by considering the photohadronic and electromagnetic interactions undergone by a population of protons and electrons accelerated in the jet. However, observations by the Pierre Auger Observatory suggest that the high-energy tail of the CR spectrum may have a component of nuclei heavier than protons, with an average mass between that of pure protons and pure iron-56 [3].

While studies like [4] have addressed the interactions of heavier nuclei in AGNs, neutrino production is treated in a simplified fashion, where the shape of the emitted neutrino spectrum is based on an analytical estimation and the and used the total observed CR energy flux to normalize the total CR emissivity of the entire AGN population. In the first part of this talk we will show that the spectrum and luminosity of the neutrino emission by blazars actually depends critically on the composition of the accelerated CRs. We therefore introduce a novel model of CR interactions in blazar jets, which allows for the acceleration of nuclei heavier than protons, and numerically calculates all photo-nuclear and photo-hadronic interactions taking place in the source [5]. By using multi-wavelength blazar observations as input to the model, the CR and neutrino spectrum emitted by the source can therefore be consistently calculated.

This single-source model of photo-hadronic interactions can be generalized and applied to the entire cosmological blazar distribution, in order to assess under what conditions this particular source class can power the entire diffuse flux of high-energy neutrinos observed by IceCube. Given the lack of statistical correlations between neutrino arrival directions and the positions of gammaray blazars, [6] have excluded the contribution of these sources to the IceCube neutrino flux at a level any higher than 20\%. However, this sample corresponds to only a fraction of the entire population of blazars, due to the limited sensitivity of current gamma-ray telescopes. Motivated by these arguments, in Section 3 we will use the blazar neutrino model to estimate the diffuse neutrino emission from the entire blazar population. The conclusion is that under certain conditions, a subset of low-luminosity blazars is able to account for the entire spectrum of astrophysical neutrinos detected by IceCube in the range from sub-PeV to PeV energies [7]. At the same time, a limit can be set to the amount of CRs accelerated in high-luminosity blazars, due to the lack of overlap between observed neutrinos and gamma-ray blazars.

\section{A model for hadro-nuclear interactions in blazar jets}

State-of-the-art lepto-hadronic models calculate the radiation processes undergone by a population of electrons and protons accelerated in the jet, interacting with a non-thermal photon field. Neutrinos are produced through inelastic interactions of the injected protons with target photons

\footnotetext{
* Speaker.
} 

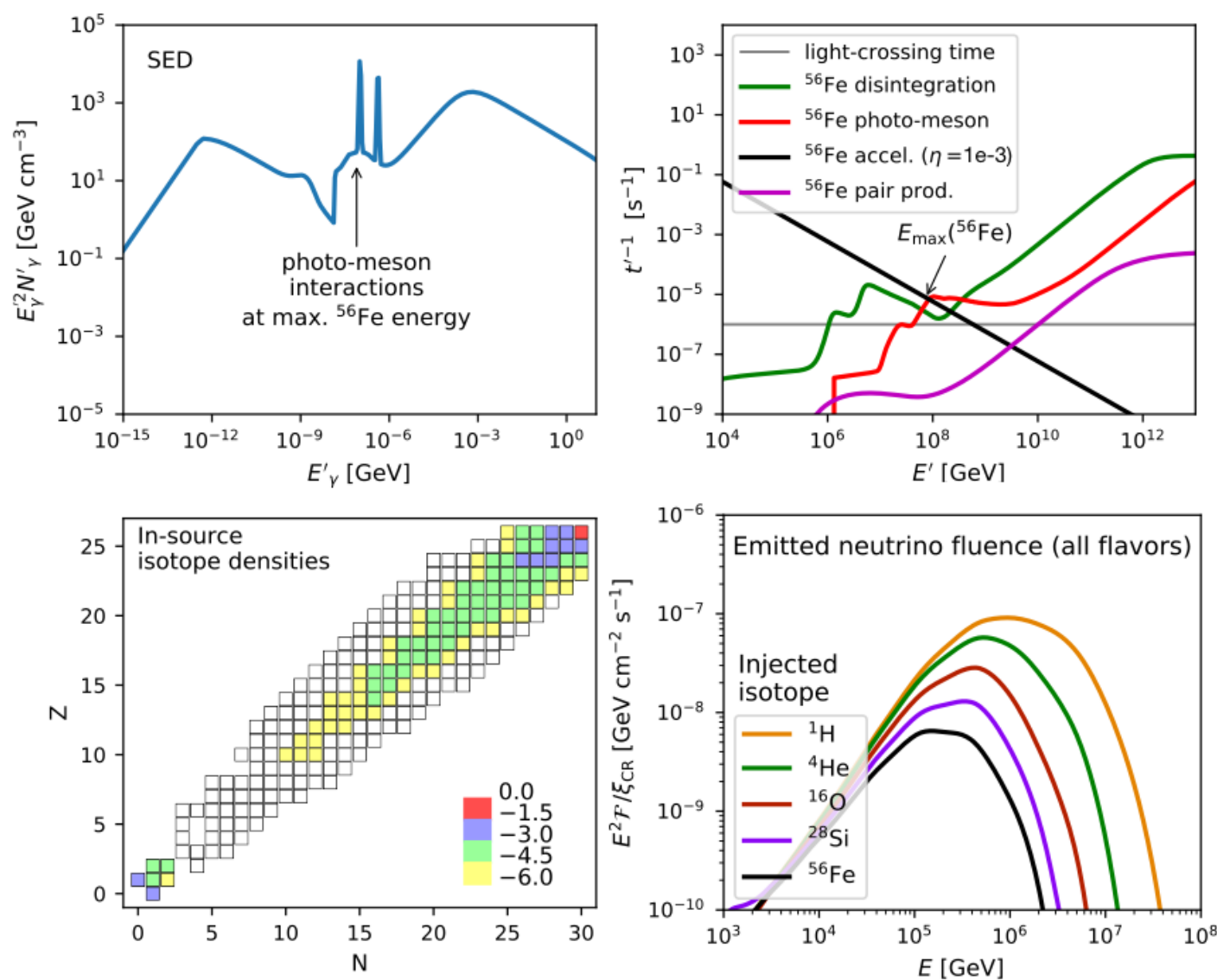

Figure 1: Results of a simulation of the photo-hadronic processes taking place in a blazar jet with luminosity $L_{\gamma}=10^{48.5} \mathrm{erg} / \mathrm{s}$, considering the acceleration of iron-56. Upper left: time-independent SED, adopted from the Fermi blazar sequence [8]. Upper right: interaction rates of relevant radiative processes. Lower left: energy densities of nuclear isotopes, normalized to the injection luminosity. Lower right: all-flavor neutrino fluence emitted during a flare for the case of different injected isotopes, from protons to iron-56. Neutrino fluences shown in the observer's frame for redshift $z=1$. Figures adapted from [5] and [7].

in the blazar jet, resulting in the production of mesons, which can subsequently decay producing neutrinos. In this model, we additionally account for the possible acceleration of nuclei heavier than protons, by including all relevant radiation processes such as photo-nuclear disintegration. This process refers to interactions undergone by nuclei below the pion production threshold, $E_{A}^{\prime \text { thresh }} \approx 0.2 \mathrm{GeV} / E_{\gamma}^{\prime 1}$. Photo-disintegration on target photons leads to the breakup of the nucleus into lighter isotopes and one or multiple lighter fragments, mostly protons and neutrons and alpha particles (see e.g. Ref. [9]). At higher energies, this process is often accompanied by photo-meson production by individual nucleons, leading to neutrino emission.

The assumptions and parameter values of the model are described in detail in [5]. We assume that CRs are accelerated in a spherical plasma blob in the jet of radius $r_{\text {blob }}^{\prime} \simeq 3 \cdot 10^{16} \mathrm{~cm}$. This size is estimated from a typical flare duration of $t_{\text {flare }}^{\prime}=10^{6} \mathrm{~s} \mathrm{such}$ that $r_{\text {blob }}^{\prime} \simeq \Gamma c t_{\text {flare }}$. The blob travels along the jet with Lorentz factor $\Gamma=10$ in a direction close to that of the observer.

\footnotetext{
${ }^{1}$ In this work, primed quantities refer to the jet rest frame and unprimed ones to the observer's frame.
} 
The transport of accelerated nuclei in the source is modeled as a system of partial differential equations. Details may be found in Ref. [10], where the same method is used for solving the transport equations based on the NeuCosmA code [11]. In this contribution, we will limit ourselves to the case of a pure injection composition, where the element can have any mass up to iron-56.

In each simulation the blazar SED is taken from observations, and we assume that it emerges self-consistently from all participating radiation processes. However, it remains important to be checked if and under what conditions this assumption can hold. The feedback of hadronic photons to the SED is non-trivial and can be difficult to compute, even in the simplest proton-only case. As shown in Fig.4 of [12], the feedback may show up in the X-ray range - the saddle of the two humps.

Based on the work of [8], the description of SEDs from observed blazars can be reasonably approximated by the so-called blazar sequence, an analytical description of the SED that evolves as a function of the blazar's gamma-ray luminosity $L_{\gamma}$. The brighter the source is in gamma rays, the "redder" the SED broadband emission (see Fig. 6 of Ref. [8] and $c f$. Fig. 2 of Ref. [7]).

The upper left panel of Fig. 1 shows an SED of a prototype from the blazar sequence with $L_{\gamma}=10^{48.5} \mathrm{erg} / \mathrm{s}$, shown as a density spectrum in the rest frame of the jet. Besides the typical double-hump structure from non-thermal processes in the jet, in the case of flat-spectrum radio quasars (FSRQs), we have additionally considered the effect of the radiation fields from structures surrounding the jet, such as thermal emission from an accretion disk and a dust torus and atomic emission from a broadline region (BLR). On the contrary, BL Lacs do not exhibit broad line emission, which may be due to a small BLR, or a radiatively inefficient accretion disk.

These external features of FSRQs can be seen in the example in Fig. 1 emerging between the two non-thermal humps of the SED. These external components are observed from the jet with an enhanced luminosity, due to the Lorentz boost between the rest frame of the black hole and that of the jet. This enhances neutrino production in the jet, as well as during CR propagation through these external fields after escaping the jet, an aspect that was also explicitly considered in this work.

The upper right panel of Fig. 1 shows the rates of the processes undergone by an iron-56 nucleus that interacts with the same photon field in the jet. The maximum energy of the injected element, $E_{i, \max }^{\prime}$, is determined self-consistently in each simulation by equating the acceleration rate, shown as a black line for the case of iron-56,

$$
t_{\mathrm{acc}}^{\prime-1}\left(E^{\prime}\right)=\frac{\eta B Z e c^{2}}{E^{\prime}}=\frac{\eta c}{R_{L}\left(E^{\prime}\right)}
$$

(where $R_{L}(E \prime)$ is the Larmor radius of the nucleus and $\eta$ is the acceleration efficiency), with the sum of synchrotron loss, photo-disintegration and photo-meson production rates, as well as the light-crossing time of the blob.

In this work, we choose $\eta=10^{-3}$ for the acceleration efficiency. This was not the case in [5], where an ultra-efficient acceleration was considered of $\eta=1$, which allows for the emission of CRs up to ultra-high energies. Such scenario would be necessary if we intended to explain the highenergy tail of the observed CR spectrum; however, the neutrinos emitted would have equivalently high energies of order $\sim \mathrm{EeV}$, which is not suitable for explaining the currently measured flux of astrophysical neutrinos, which extends up to no more than a few PeV. Furthermore, there is 
evidence that relativistic shocks may in fact be inefficient accelerators through diffusive shock acceleration [13], which supports low values of the acceleration efficiency parameter.

We can see that in this example the maximum energy of iron-56 nuclei is limited by photomeson production (red curve in the upper left panel). However, at most energies photo-disintegration (green curve) dominates over this photo-meson production, as has already been discussed e.g. in Ref [4].

The high rates of photo-disintegration of heavy elements limits their maximum energy, and therefore neutrino production through photo-meson production. This effect can be seen in the lower right panel of Fig. 1, where the emitted neutrino spectrum is shown to have a suppressed maximum energy and total fluence the heavier the injected isotope. Note that this is a direct consequence of the way the maximum energy of the injected isotope is calculated, discussed above. Therefore, in this model a pure injection composition of protons corresponds to the most optimistic scenario for neutrino production in blazars.

\section{Diffuse neutrino flux from the entire blazar population}

Based on this model, we can study the neutrino emissivity of blazars across a wide range of luminosities, where in each luminosity bin following the prototypes from the blazar sequence: from low-luminosity, high-frequency peaked BL Lacs (HBLs) to high-luminosity, low-frequency peaked BL Lacs (LBLs), as well as FSRQs. This will allow us to use the model described above to assess the entire blazar population as a potential source of the observed diffuse IceCube neutrino flux.

The neutrino emission efficiency of each individual source, $\varepsilon_{v}$, can be defined as the ratio between the neutrino luminosity of the source and the luminosity in injected CRs, $\varepsilon_{v}=L_{v} / L_{\mathrm{CR}}$. We obtain this parameter from the source model presented previously, assuming the most optimistic case of pure proton injection. The baryonic loading $\xi_{\mathrm{CR}}$ (defined as the ratio of CR to gammaray luminosity, $\xi_{\mathrm{CR}}=L_{\mathrm{CR}} / L_{\gamma}$ ) will be allowed to vary for blazars of different luminosities. We will show that low-luminosity blazars can provide the dominant contribution to the high-energy neutrinos with energy between a few hundreds of $\mathrm{TeV}$ and a few $\mathrm{PeV}$, while the contribution of very bright sources to the neutrino flux must be highly suppressed in order to respect the blazar stacking limit [6]. We also discuss explicitly the role of TXS 0506+056, and what we can learn from the population study for future such observations.

Considering the high-energy starting events (HESE) in IceCube, the contribution of resolved blazars to the astrophysical neutrino flux cannot be larger than $\sim 20 \%-25 \%$ [14] based on the missing association with sources in $\gamma$-ray catalogs, or even less based on theoretical considerations [15]. On the other hand, there have been indications of associations of individual neutrino events with AGNs [16, 17, 18], which means that it is plausible that a few of IceCube's events stem from resolved blazars. More recently, direct evidence has been found of a correlation between IceCube neutrinos and the object TXS 0506+056 [19,20], which represents a breakthrough discovery in multi-messenger astrophysics. This observation may support the blazar origin of the diffuse neutrino flux, which may be mostly powered by unresolved objects. 

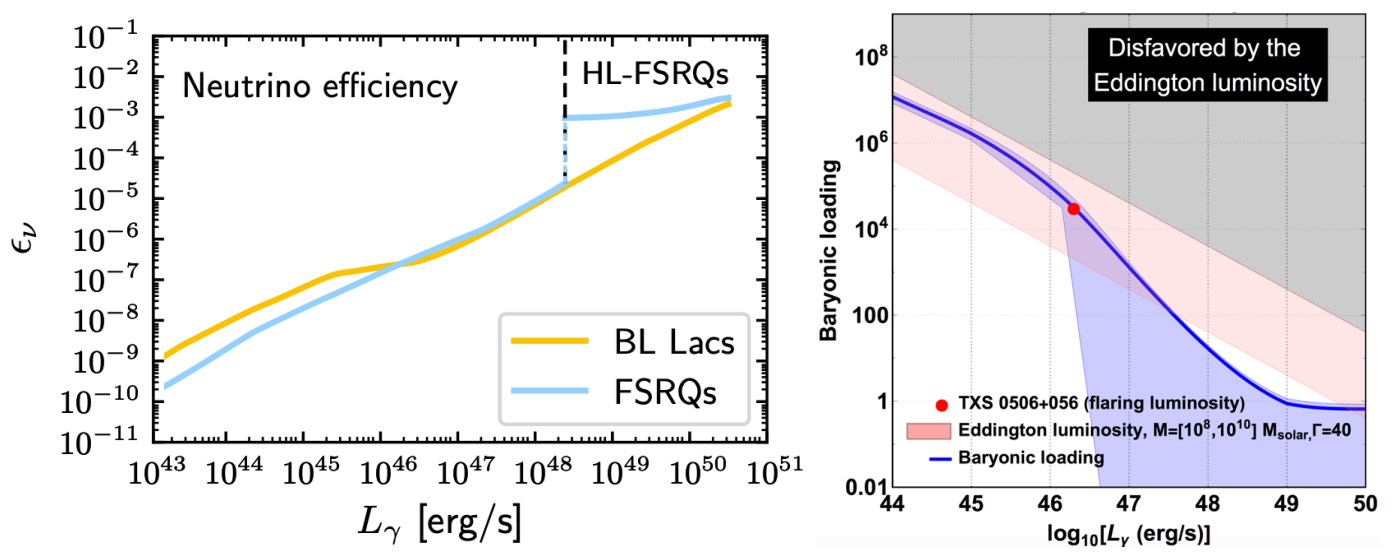

Figure 2: Left: Neutrino production efficiency of BL Lacs (yellow) and FSRQs (blue) obtained from the numerical blazar model a function of the gamma-ray luminosity of the source. Right: baryonic loading of blazars obtained in order to explain the sub-PeV to $\mathrm{PeV}$ diffuse IceCube flux, as a function of $L_{\gamma}$. The pink band represents the Eddington luminosity, assuming $\Gamma_{\mathrm{b}}=10-40$ and a black hole mass of $10^{7.5}-10^{9.5} M_{\odot}$. The gray region is disfavored, since the proton power would exceed the source's Eddington luminosity. Figures adapted from [7].

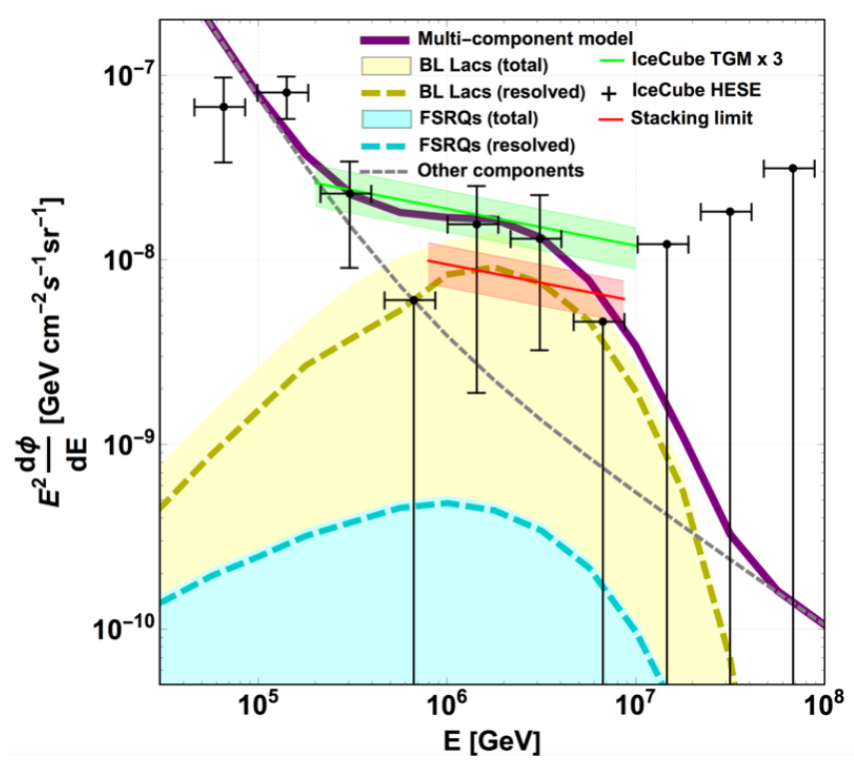

Figure 3: Diffuse neutrino spectrum in IceCube estimated for the baryonic loadings shown in Fig. 2. The black points represent the all-flavor fluxes of IceCube high-energy starting events (HESE, [21]), the green line represents the throughgoing muon flux (multiplied by 3 to account for all three neutrino flavors, [22]) and the red line represents the IceCube stacking limit on the blazar contribution [6]. The shaded yellow and cyan represent the contribution of BL Lacs and FSRQs, respectively, to the total flux of neutrinos, whereas the dotted yellow and cyan curves denote the contribution from resolved sources only. The purple solid curve represents the total neutrino flux expected from blazars. In this case most of the flux is powered by resolved sources, particularly FSRQs. 


\section{References}

[1] ICECUBE collaboration, Evidence for High-Energy Extraterrestrial Neutrinos at the IceCube Detector, Science 342 (2013) 1242856 [1311.5238].

[2] ICECube Collaboration collaboration, First observation of PeV-energy neutrinos with IceCube, Phys.Rev.Lett. 111 (2013) 021103 [1304.5356].

[3] PIERre Auger collaboration, Combined fit of spectrum and composition data as measured by the Pierre Auger Observatory, JCAP 1704 (2017) 038 [1612.07155].

[4] L. A. Anchordoqui, D. Hooper, S. Sarkar and A. M. Taylor, High-energy neutrinos from astrophysical accelerators of cosmic ray nuclei, Astropart. Phys. 29 (2008) 1 [astro-ph/ 0703001 ].

[5] X. Rodrigues, A. Fedynitch, S. Gao, D. Boncioli and W. Winter, Neutrinos and Ultra-High-Energy Cosmic-Ray Nuclei from Blazars, Astrophys. J. 854 (2018) 54 [1711.02091].

[6] ICECUBE collaboration, The contribution of Fermi-2LAC blazars to the diffuse TeV-PeV neutrino flux, Astrophys. J. 835 (2017) 45 [1611.03874].

[7] A. Palladino, X. Rodrigues, S. Gao and W. Winter, Interpretation of the diffuse astrophysical neutrino flux in terms of the blazar sequence, Astrophys. J. 871 (2019) 41 [1806.04769].

[8] G. Ghisellini, C. Righi, L. Costamante and F. Tavecchio, The Fermi blazar sequence, Mon. Not. Roy. Astron. Soc. 469 (2017) 255 [1702.02571].

[9] D. Boncioli, A. Fedynitch and W. Winter, Nuclear Physics Meets the Sources of the Ultra-High Energy Cosmic Rays, Scientific Reports 7 (2017) 4882 [1607. 07989 ].

[10] D. Biehl, D. Boncioli, A. Fedynitch and W. Winter, Cosmic-Ray and Neutrino Emission from Gamma-Ray Bursts with a Nuclear Cascade, 1705.08909.

[11] P. Baerwald, S. Hümmer and W. Winter, Systematics in the Interpretation of Aggregated Neutrino Flux Limits and Flavor Ratios from Gamma-Ray Bursts, Astropart. Phys. 35 (2012) 508 [1107.5583].

[12] S. Gao, M. Pohl and W. Winter, On the direct correlation between gamma-rays and PeV neutrinos from blazars, Astrophys. J. 843 (2017) 109 [1610.05306].

[13] Y. Inoue and Y. T. Tanaka, Baryon Loading Efficiency and Particle Acceleration Efficiency of Relativistic Jets: Cases For Low Luminosity BL Lacs, Astrophys. J. 828 (2016) 13 [1603. 07623 ].

[14] ICECUBE collaboration, The contribution of Fermi-2LAC blazars to the diffuse TeV-PeV neutrino flux, Astrophys. J. 835 (2017) 45 [1611.03874].

[15] A. Palladino and F. Vissani, Can BL Lacertae emission explain the neutrinos above 0.2 PeV?, Astron. Astrophys. 604 (2017) A18 [1702.08779].

[16] E. Resconi, S. Coenders, P. Padovani, P. Giommi and L. Caccianiga, Connecting blazars with ultrahigh-energy cosmic rays and astrophysical neutrinos, Mon. Not. Roy. Astron. Soc. 468 (2017) 597 [1611.06022].

[17] P. Padovani, E. Resconi, P. Giommi, B. Arsioli and Y. L. Chang, Extreme blazars as counterparts of IceCube astrophysical neutrinos, Mon. Not. Roy. Astron. Soc. 457 (2016) 3582 [1601. 06550 ].

[18] M. Kadler et al., Coincidence of a high-fluence blazar outburst with a PeV-energy neutrino event, Nature Phys. 12 (2016) 807 [1602.02012]. 
[19] Liverpool Telescope, MAGiC, H.E.S.S., AGILE, Kiso, VLA/17B-403, INTEGRAL, KAPTEYN, SubARU, HAWC, FERmi-LAT, ASAS-SN, VERITAS, KANATA, ICECUbE, SWIFT NUSTAR collaboration, Multimessenger observations of a flaring blazar coincident with high-energy neutrino IceCube-170922A, Science 361 (2018) eaat1378 [1807.08816].

[20] ICECUBE collaboration, Neutrino emission from the direction of the blazar TXS 0506+056 prior to the IceCube-170922A alert, Science 361 (2018) 147 [1807.08794].

[21] ICECUBE collaboration, A combined maximum-likelihood analysis of the high-energy astrophysical neutrino flux measured with IceCube, Astrophys. J. 809 (2015) 98 [1507.03991].

[22] ICECUBE collaboration, Observation and Characterization of a Cosmic Muon Neutrino Flux from the Northern Hemisphere using six years of IceCube data, Astrophys. J. 833 (2016) 3 [1607. 08006 ]. 